\title{
DIMENSÕES DE PERSONALIDADE, QUALIDADE DE VIDA E DEPRESSÃO EM IDOSAS
}

\author{
Tatiana Quarti Irigaray* \\ Rodolfo Herberto Schneider
}

RESUMO. Os aspectos de personalidade têm sido apontados como fatores que podem contribuir para a avaliação da qualidade de vida e o desencadeamento de depressão na velhice. O objetivo deste estudo foi investigar a relação entre fatores de personalidade, qualidade de vida e depressão em idosas. Utilizou-se o método amostral de conveniência. Cento e três idosas foram avaliadas e responderam sobre condições sociodemográficas, aspectos de personalidade (IFP), qualidade de vida (WHOQOL-bref) e depressão (GDS). De modo geral, essas mulheres apresentaram características medianas para todos os fatores de personalidade, tendo como dimensões mais referidas a alta deferência e baixa agressão. As participantes deste estudo não apresentaram como característica a presença de depressão. O estudo aponta para a existência de associação entre dimensões de personalidade, qualidade de vida e depressão em idosas; contudo, outros estudos são sugeridos.

Palavras-chave: Personalidade; qualidade de vida; depressão em idosas.

\section{DIMENSIONS OF PERSONALITY, QUALITY OF LIFE AND DEPRESSION IN ELDERLY WOMEN}

\begin{abstract}
Personality aspects have been indicated as factors that may contribute to the quality of life assessment and the onset of depression in old age. The purpose of this study is to investigate the relationship among the personality factors, the quality of life and depression in the elderly. The sampling process used was the convenience method. One hundred three elderly women were assessed and asked about sociodemographic conditions, personality aspects (PFI), quality of life (WHOQOL-bref) and depression (GDS). In general terms, the elderly women in this sample, showed median characteristics for all the PFI factors, having the aspects of high deference and low aggression been the most cited. The participants in this study did not show the presence of depression as a characteristic. The study highlights the existence of an association among the personality dimensions, the quality of life and depression in the elderly. Nevertheless, further studies are suggested.
\end{abstract}

Key words: Personality; quality of life; depression in elderly women.

\section{DIMENSIONES DE PERSONALIDAD, CALIDAD DE VIDA Y DEPRESIÓN EN ANCIANAS}

RESUMEN. Los aspectos de personalidad han sido señalados como factores que contribuyen para la evaluación de calidad de vida y desencadenamiento de depresión en la vejez. El objetivo de este estudio fue investigar la relación entre factores de personalidad, calidad de vida y depresión en ancianas. Se utilizó el método de muestra de conveniencia. Ciento tres ancianas fueron evaluadas y contestaron sobre condiciones sociodemográficas, aspectos de personalidad (IFP), calidad de vida (WHOQOL-bref) y depresión (GDS). Por regla general, las ancianas presentaron dimensiones medianas para todos los factores de personalidad, teniendo como dimensiones más referidas la alta diferencia y baja agresión. Las participantes no presentaron depresión. El estudio señala para la existencia de una asociación entre dimensiones de personalidad, calidad de vida y depresión en ancianas. Sin embargo, otros estudios son sugeridos.

Palabras clave: Personalidad; calidad de vida; depresión en ancianas.

O envelhecimento é um processo vivido por cada indivíduo de maneira diferente, sofrendo influência de fatores históricos, sociais, culturais, socioeconômicos, educacionais e intelectuais, de aspectos de personalidade e da presença ou não de doenças. Não é uma só a definição do termo personalidade, mas varia de acordo com a teoria de personalidade empregada. Segundo a teoria de Murray, a personalidade refere-se a uma série de eventos que abrangem toda a vida do indivíduo, refletindo elementos duradouros e

* Instituto de Geriatria e Gerontologia da Pontifícia Universidade Católica do Rio Grande do Sul-PUCRS. 
recorrentes do comportamento, bem como elementos novos e únicos, portanto, é o agente organizador ou governador do indivíduo (Hall, Lindzey \& Campbell, 2000).

Existem inúmeras teorias que tentam explicar o desenvolvimento da personalidade na velhice. As primeiras pesquisas sugeriram que a personalidade se tornava mais rígida com o envelhecimento e que se desenvolvia muito pouco na velhice (Papalia \& Olds, 2000; Tavares, 2005). Para Neugarten, Moore e Lowe (1965), as pessoas se tornavam mais introvertidas à medida que envelheciam.

Os estudos que surgiram a partir da década de 70 sugerem o contrário: ocorreria uma estabilidade dos traços de personalidade ao longo da vida adulta e da velhice (Costa, Herbst, McCrae \& Siegler, 2000; Herbst, McCrae, Costa, Feaganes, \& Siegler, 2000; McCrae \& Costa Jr., 1987, 1994). De acordo com esses estudos, a personalidade não se tornaria mais rígida, mas permaneceria da mesma forma como sempre fora desde a vida adulta.

Alguns autores defendem a ideia de que diferenças entre coortes se refletem na personalidade de idosos (Ruth \& Coleman, 1996; Schaie \& Willis, 1991). Para Schaie e Willis, as pessoas de coortes mais recentes apresentam maior flexibilidade, isto é, menor rigidez que as de coortes anteriores. Tais diferenças, provavelmente, estão associadas à influência de fatores sócio-históricoculturais das gerações mais antigas. Segundo Ruth e Coleman (1996), as diferenças de personalidade entre jovens, adultos e idosos são causadas por diversidades entre as gerações (coortes), e não por distinções de idade.

Teorias atuais de personalidade indicam que, apesar da aparente estabilidade da personalidade na idade adulta, existe considerável potencial para mudança (Field \& Millsap, 1991; Helson, Jones \& Kwan, 2002; LabouvieVief, Diehl, Tarnowski \& Shen, 2000; Maiden, Peterson, Caya \& Hayslip Jr., 2003; McAdams, 1995). O estudo de Maiden et al. constatou que os traços de personalidade são modificáveis na velhice, principalmente porque nessa fase de vida as pessoas têm maior probabilidade de serem confrontadas com eventos de vida que requerem adaptação. Embora muitos indivíduos possam manter a personalidade estável, sob circunstâncias de vida normais, especula-se que diante de situações mais complexas a personalidade mudaria para se adaptar às mudanças da vida.

Conforme algumas pesquisas, as dimensões de personalidade podem estar relacionadas aos índices de resiliência e bem-estar subjetivo (Ryff, 1991; Staudinger, Marsiske \& Baltes, 1993), bem como ao desencadeamento de sintomatologia depressiva (Costa et al., 2000; Costa, McCrae \& Zonderman, 1987; Diener \&
Diener, 1996; Martin, Valora \& Poon, 2002; Small, Herzog, Hultsch \& Dixon, 2003; Steunenberg, Beekman, Deeg \& Kerkhof, 2006; Watson \& Walker, 1996). O bem-estar das pessoas tem sido considerado por alguns autores como sinônimo de qualidade de vida (Kahn \& Thomas, 2002).

Para a Organização Mundial de Saúde (The WHOQOL group, 1998), o conceito de qualidade de vida é definido como a percepção que o indivíduo tem sobre a sua posição na vida, no contexto de sua cultura e dos sistemas de valores da sociedade em que vive, e também em relação aos seus objetivos, expectativas, padrões e preocupações. Segundo Neri (2000), o conceito de qualidade de vida na velhice é resultante da atuação de eventos concorrentes múltiplos, referindo-se à adaptação de indivíduos e grupos de pessoas em diferentes épocas da vida de uma ou várias sociedades.

Uma estreita relação entre qualidade de vida e intensidade de sintomas depressivos e/ou níveis de desesperança no idoso foi encontrada no estudo de Trentini (2004). Para a autora, o indivíduo que avalia a sua qualidade de vida como negativa apresenta significativamente mais sintomas depressivos em relação aos que têm esperança, os quais, segundo alguns estudos, apresentam melhores condições de saúde (Carpenito 1995; Farran, Herth \& Popovich, 1995). Outros estudos também encontraram associação entre má qualidade de vida e depressão (Herman et al., 2002; Kuehner, 2002; Xavier et al., 2002).

$\mathrm{Na}$ velhice, conforme inúmeros autores, a personalidade atua como um dos principais fatores desencadeantes de sintomatologia depressiva (Martin et al., 2002; Small et al., 2003; Steunenberg et al., 2006; Watson \& Walker, 1996). Segundo Steunenberg et al., a personalidade está mais fortemente relacionada ao início da depressão do que a recursos sociais e ao estado de saúde.

$\mathrm{O}$ presente artigo teve por objetivo investigar a relação entre fatores de personalidade, qualidade de vida e depressão em mulheres idosas de um grupo de convivência da terceira idade de uma universidade. Também buscou avaliar dimensões gerais de personalidade, intensidade e incidência de sintomatologia depressiva e aspectos de qualidade de vida na amostra estudada.

\section{MÉTODOS}

\section{Participantes}

A amostra foi composta por 103 idosas, com idade mínima de sessenta anos. Os sujeitos foram recrutados em um grupo de convivência da terceira idade de uma 
universidade e tinham escolaridade mínima de sete anos de estudo. As participantes eram idosas independentes, residiam na comunidade e frequentavam este grupo para realizar atividades cognitivas e sociais. $O$ grupo é composto, em sua grande maioria, por mulheres idosas, apresentando, em média, 150 participantes por semestre. $\mathrm{O}$ processo de amostragem foi o de conveniência.

\section{Instrumentos}

Os instrumentos utilizados foram: ficha de dados sociodemográficos, Inventário Fatorial de Personalidade - IFP (Pasquali, Azevedo \& ghesti, 1997), WHOOQOL - Bref (Fleck et al., 1999 e Escala de Depressão Geriátrica - GDS - 15 (Sheikh \& Yesavage, 1986).

1. A ficha de dados sociodemográficos incluiu as seguintes variáveis: sexo, idade, estado civil, escolaridade, renda, situação de moradia, ocupação anterior, ocupação atual, percepção de saúde, uso de medicação, realização de atividade física e tempo de participação no grupo de convivência.

2. O Inventário Fatorial de Personalidade - IFP (Pasquali, Azevedo \& Ghesti, 1997) é um inventário da personalidade objetivo, que avalia 15 necessidades ou motivos psicológicos (assistência, dominância, ordem, denegação, intracepção, desempenho, exibição, heterossexualidade, afago, mudança, persistência, agressão, deferência, autonomia e afiliação).

3. WHOQOL - bref (Fleck \& et al., 1999) é uma versão abreviada do instrumento WHOQOL-100, que foi desenvolvido pelo grupo de qualidade de vida da Organização Mundial da Saúde. Avalia quatro domínios (físico, psicológico, relações sociais e meio ambiente), incluindo questões de avaliação global de qualidade de vida.

4. A Escala de Depressão Geriátrica - GDS-15 (Sheikh \& Yesavage, 1986) é uma medida utilizada para o rastreamento de depressão em idosos. A versão curta é composta por 15 perguntas e respostas classificadas em sim ou não.

\section{Procedimentos}

\section{Procedimentos para coleta dos dados}

Foram realizados contatos com o grupo de convivência, procedendo-se, assim, à inclusão das participantes na amostra. Todas as idosas preencheram o Termo de Consentimento Livre e Esclarecido e, a partir daí, foram convidadas a responder acerca de informações sociodemográficas, fatores de personalidade (Pasquali, Azevedo \& Ghesti, 1997), aspectos de qualidade de vida
(Fleck et al., 1999) e sintomatologia de depressão (Sheikh \& Yesavage, 1986).

Tanto a ficha de dados sociodemográficos quanto o IFP, o WHOQOL-bref e a GDS são de caráter autoadministrável, contudo um auxiliar de pesquisa (estudante de Psicologia) sempre esteve à disposição das idosas para qualquer esclarecimento. As aplicações dos instrumentos foram feitas em grupos de até 15 participantes.

O projeto foi devidamente examinado e aprovado pelo Comitê de Ética em Pesquisa sob o n. ${ }^{\circ}$ 06/0309.

\section{Procedimentos para análise dos dados}

A descrição das variáveis foi realizada por meio das frequências absolutas e relativas e da média e desvio padrão. As associações entre os fatores de personalidade do IFP e os domínios de qualidade de vida (WHOQOLbref) foram medidas pelo coeficiente de Correlação de Pearson, uma vez que os dados satisfaziam as condições para uso de um teste paramétrico. De forma diferente, utilizou-se o Coeficiente de Correlação de Spearman para calcular a associação entre os fatores de personalidade do IFP e a variável depressão (GDS), pois as variáveis analisadas não satisfaziam as condições para uso de um teste paramétrico. O Teste-t de Student foi utilizado para a comparação realizada entre as variáveis depressão (GDS) e os domínios de qualidade de vida e QV geral (WHOQOL-bref) e os clusters do IFP. Foram consideradas significativas as associações com valores de $\mathrm{p}<0,05$. Para análise dos dados, foi utilizado o programa SPSS para ambiente Windows, versão 13.0.

Os clusters foram agrupados segundo o método de agrupamento (clustering) hierárquico. No cluster 1 , foram agrupados os indivíduos que apresentavam características de personalidade relacionadas entre si mas que se distanciavam das características dos indivíduos do cluster 2, demonstrando fatores de personalidade diferentes. Assim, separaram-se os grupos em cluster 1 e cluster 2, reunindo as dimensões de personalidade que estavam relacionadas entre si através de suas relações de similiaridades em um grupo (cluster 1) e em outro (cluster 2) as relações de dissimilaridades em relação ao primeiro. O cluster 1 foi formado por pessoas com características de personalidade mais voltadas para o outro, mais deferentes, organizadas, persistentes e interativas. De forma diferente, o cluster 2 foi composto por pessoas mais voltadas para si próprias, menos interativas e pouco dominantes.

$\mathrm{O}$ teste $\mathrm{F}$ foi usado, conforme mostra a Tabela 2, somente para fins descritivos, pois os clusters foram utilizados para maximizar as diferenças entre os grupos constrastantes. Os níveis de significância observados não são corrigidos e, por isso, não podem 
ser interpretados como um teste da hipótese que os clusters são iguais. Já na Tabela 3, utilizou-se o teste t, uma vez que se estava analisando a diferença entre a média do cluster 1 e do cluster 2 em relação aos domínios de qualidade de vida (WHOQOL-bref) e a variável depressão (GDS).

\section{RESULTADOS}

A amostra contou com 103 mulheres idosas na faixa etária entre 60 e 86 anos (idade média de 69,2 $D P=6,46)$. O estado civil mais frequente entre elas foi o de viúva (44,7\%); a escolaridade prevalecente foi o ensino superior $(40,8 \%)$, e a renda mais presente foi de 6 a 10 salários mínimos (38,8\%). Quanto à situação de moradia, predominou a moradia com a família $(52,4 \%)$, a profissão de professora foi a ocupação anterior mais frequente $(28,1 \%)$ e a aposentadoria a condição atual prevalente $(73,8 \%)$. A percepção de saúde predominante foi a de saudável $(81,6 \%)$, o uso de medicação $(89,3 \%)$ e a realização de atividade física $(74,8 \%)$ também foram bastante citados entre as idosas. A maior parte delas $(62,1 \%)$ participa do grupo de convivência há mais de um ano.

Na tabela 1 são apresentadas as médias e os desvios padrão dos fatores do IFP, dos domínios de qualidade de vida (WHOQOL-bref) e da GDS.

Tabela 1 Descrição dos Fatores do IFP, do WHOQOLbref (QV) e da GDS.

\begin{tabular}{lcc}
\hline Variáveis & $\overline{\mathrm{X}}$ & $\mathrm{DP}$ \\
\hline Fatores IFP & & \\
Assistência & 51,59 & 7,04 \\
Intracepção & 44,69 & 7,78 \\
Afago & 45,84 & 8,74 \\
Deferência & 50,68 & 6,42 \\
Afiliação & 55,06 & 6,64 \\
Dominância & 30,31 & 8,64 \\
Denegação & 37,98 & 6,94 \\
Desempenho & 47,17 & 7,49 \\
Exibição & 28,61 & 8,46 \\
Agressão & 24,58 & 7,53 \\
Ordem & 50,17 & 8,07 \\
Persistência & 45,90 & 8,74 \\
Mudança & 47,74 & 7,41 \\
Autonomia & 45,04 & 7,08 \\
QV & & \\
Domínio Físico & 71,08 & 14,39 \\
Domínio Psicológico & 63,19 & 10,32 \\
Domínio Relacionamentos Sociais & 68,77 & 16,65 \\
Domínio Meio Ambiente & 74,39 & 11,33 \\
QV Geral & 75,60 & 16,07 \\
GDS & 2,65 & 2,42 \\
\hline
\end{tabular}

Em relação aos fatores do IFP, entre os domínios de qualidade de vida (WHOQOL-bref) e a variável depressão (GDS) foram encontradas correlações significativas. Por meio do Coeficiente de Correlação de Pearson obteve-se uma correlação negativa e fraca, porém significativa entre o fator afago do IFP e o domínio físico do WHOQOL-bref ( $\mathrm{r}=-0,201 ; p<0,05) ; p<0,05)$ (WHOQOL-bref). Foram encontradas correlações fracas, porém significativas, entre o domínio psicológico do WHOQOL-bref e os fatores de personalidade afiliação $(\mathrm{r}=0,251 ; p<0,05)$, persistência $(\mathrm{r}=0,204 ; p<0,05)$ e mudança $(\mathrm{r}=0,286 ; p<0,05)$.

Para o domínio relacionamentos sociais (WHOQOLbref) e os fatores do IFP foi obtida uma única correlação significativa positiva, embora fraca, com o fator mudança $(\mathrm{r}=0,206 ; p<0,05)$. Para o domínio meio ambiente (WHOQOL-bref) foram encontradas correlações significativas positivas, apesar de fracas, entre os fatores ordem $(\mathrm{r}=0,234 ; \quad p<0,05)$ e persistência $(\mathrm{r}=0,234$; $p<0,05)$. Por meio do Coeficiente de Correlação de Spearman foram obtidas correlações significativas, apesar de fracas, entre a variável depressão (GDS) e os fatores do IFP afago $(\mathrm{r}=0,212 ; p<0,05)$ e agressão $(\mathrm{r}=0,234$; $p<0,268$ ), e uma correlação negativa significativa ( $\mathrm{r}=-$ $0,212 ; p<0,05)$, embora fraca, entre o fator persistência do IFP e a variável depressão (GDS).

No intuito de melhor explorar os aspectos de personalidade foi realizada uma análise de clusters (Tabela 2). Os clusters foram usados para maximizar as diferenças entre os casos em diferentes agrupamentos. Conforme pode ser verificado, o cluster 1 é formado por pessoas mais voltadas para o cuidado dos outros, interessadas no outro, mais deferentes, organizadas, persistentes e interativas; já o cluster 2 é composto por pessoas mais voltadas para si próprias, menos interativas e pouco dominantes. A Figura 1 mostra os clusters obtidos através do agrupamento das dimensões de personalidade do Inventário Fatorial de Personalidade (cluster 1 e cluster 2).

Tabela 2 - Análise de clusters para o IFP

\begin{tabular}{lcccc}
\hline \multirow{2}{*}{ Fatores IFP } & $\begin{array}{c}\text { Cluster 1 } \\
(\mathbf{n = 6 1})\end{array}$ & $\begin{array}{c}\text { Cluster 2 } \\
(\mathbf{n = 4 2})\end{array}$ & $\mathbf{F}$ & $\mathbf{p}$ \\
\cline { 2 - 3 } & $\overline{\mathrm{X}}$ & $\overline{\mathrm{X}}$ & & \\
\hline Assistência & 53,93 & 48,19 & 19,54 & $<0,001^{*}$ \\
Intracepção & 48,28 & 39,48 & 45,90 & $<0,001^{*}$ \\
Afago & 49,70 & 40,24 & 40,42 & $<0,001^{*}$ \\
Deferência & 53,28 & 46,90 & 31,96 & $<0,001^{*}$ \\
Afiliação & 57,67 & 51,26 & 29,61 & $<0,001^{*}$ \\
Dominância & 33,80 & 25,24 & 31,74 & $<0,001^{*}$ \\
Denegação & 39,51 & 35,76 & 7,70 & $0,007^{*} *$ \\
Desempenho & 49,98 & 43,07 & 26,53 & $<0,001^{*}$ \\
Exibição & 32,49 & 22,98 & 45,08 & $<0,001^{*}$ \\
Agressão & 27,64 & 20,14 & 32,17 & $<0,001^{*}$ \\
Ordem & 53,59 & 45,21 & 35,92 & $<0,001^{*}$ \\
Persistência & 49,46 & 40,74 & 32,37 & $<0,001^{*}$ \\
Mudança & 50,16 & 44,24 & 18,63 & $<0,001^{*}$ \\
Autonomia & 47,20 & 41,93 & 15,76 & $<0,001^{*}$ \\
\hline
\end{tabular}

* significativo para $\mathrm{p}<0,05$ 


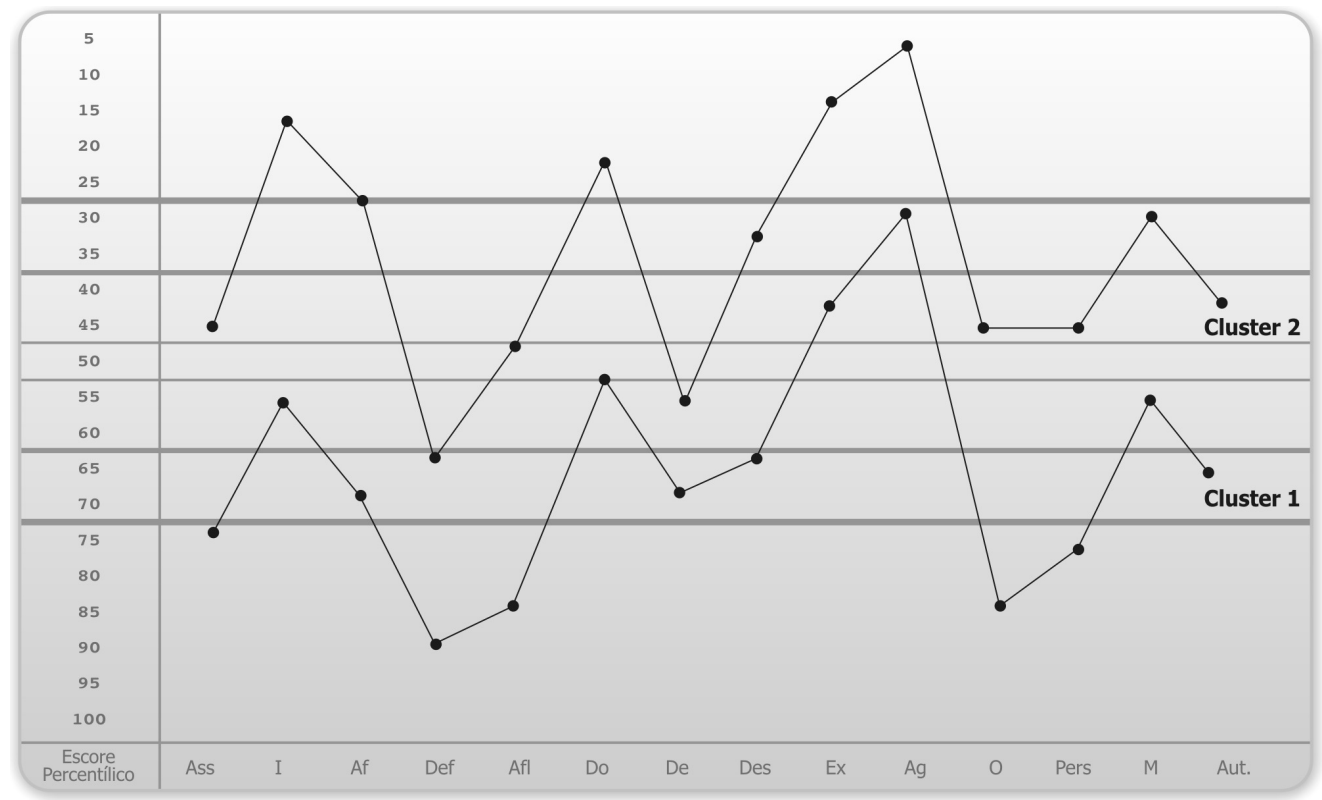

Figura 1. Média dos clusters dos fatores do Inventário Fatorial de Personalidade.

Na tabela 3 estão apresentados os resultados da comparação entre os domínios de qualidade de vida (WHOQOL-bref), a variável depressão (GDS) e os clusters do IFP. Por meio do Teste-t de Student foi possível verificar uma diferença significativa para a variável depressão (GDS) nos clusters 1 e 2. Conforme essa comparação, as pessoas agrupadas no cluster 1 apresentam mais sintomas de depressão em relação àquelas do cluster 2. Para os domínios de qualidade de vida avaliados não foram encontradas diferenças significativas entre os clusters, porém houve uma tendência à significância no domínio físico, em que a média do cluster 2 foi mais alta.

Tabela 3. Comparação Entre os Domínios de Qualidade de Vida (WHOQOL-bref) e a Variável Depressão (GDS) e os Clusters do IFP

\begin{tabular}{|c|c|c|c|c|c|c|}
\hline \multirow[b]{2}{*}{ Variáveis } & \multicolumn{2}{|c|}{$\begin{array}{c}\text { Cluster } 1 \\
(N=61)\end{array}$} & \multicolumn{2}{|c|}{$\begin{array}{c}\text { Cluster } 2 \\
(N=42)\end{array}$} & \multirow[b]{2}{*}{$\mathbf{t}$} & \multirow[b]{2}{*}{$\mathbf{p}$} \\
\hline & $\overline{\bar{X}}$ & $\mathrm{DP}$ & $\overline{\mathrm{X}}$ & DP & & \\
\hline \multicolumn{7}{|l|}{$\overline{\mathbf{Q V}}$} \\
\hline Domínio Físico & 68,91 & 15,43 & 74,23 & 12,20 & $-1,87$ & 0,06 \\
\hline Domínio Psicológico & 62,98 & 10,73 & 63,49 & 9,81 & $-0,25$ & 0,80 \\
\hline $\begin{array}{l}\text { Domínio Relacionamentos } \\
\text { Sociais }\end{array}$ & 67,08 & 18,41 & 71,23 & 13,55 & $-1,32$ & 0,19 \\
\hline Domínio Meio Ambiente & 73,82 & 12,63 & 75,22 & 9,21 & $-0,65$ & 0,51 \\
\hline QV Geral & 74,59 & 17,97 & 77,08 & 12,92 & $-0,82$ & 0,41 \\
\hline GDS & 3,11 & 2,76 & 1,98 & 1,64 & $-2,61$ & $0,01 *$ \\
\hline
\end{tabular}

* significativo para $\mathrm{p}<0,05$

\section{DISCUSSÃo}

O grupo de idosas investigado apresentou, de modo geral, características medianas para todos os fatores de personalidade, tendo como dimensões mais referidas a alta deferência e baixa agressão. Deferência tem o sentido de serem pessoas caracterizadas pelo respeito, admiração e reverência, que gostam de admirar e dar suporte a um indivíduo que consideram como superior, gostando de elogiá-lo e honrá-lo, bem como de imitá-lo e obedecer-lhe. Já a agressão não é uma característica marcante desse grupo de idosas, pois elas não são caracterizadas pela raiva, irritação e ódio, nem possuem o desejo de superar com vigor a oposição. A luta, a briga, a oposição, a censura e o ataque aos outros não caracterizam o grupo avaliado. Uma possível explicação para este achado seria o fato de as idosas desta amostra fazerem parte de uma coorte que foi educada essencialmente para desempenhar papéis femininos tradicionais, tais como os de dona de casa, mãe e esposa.

Bassanezi (1997) observou que o instinto materno, a pureza, a resignação e a doçura caracterizavam as mulheres dos Anos Dourados, e que estas são as idosas de hoje. O marido e os filhos deveriam ser o centro de suas preocupações, sua felicidade vinha como consequência de um marido satisfeito. Neri (2001) descreve as mulheres como menos agressivas, mais solidárias, mais sugestionáveis, mais envolvidas e mais relacionadas socialmente do que os homens em todas as idades.

A presença de depressão não se mostrou como uma característica do grupo estudado, já que a média geral na GDS indicou ausência de sintomatologia depressiva. Outros estudos, à disposição na literatura, corroboram tais achados, defendendo a hipótese de 
associação entre a participação em uma universidade para a terceira idade e aumento do conhecimento e do contato social, e uma menor intensidade de sintomas depressivos (Cachioni, 1998; Erbolato, 1996; Loures \& Gomes, 2006; Silva, 1999).

O estudo de Loures e Gomes (2006) encontrou uma associação entre menor intensidade de depressão e participação em uma universidade aberta à terceira idade, relacionando esta menor presença de sintomas depressivos à frequência ao curso, que, provavelmente, fez com que os idosos se sentissem menos abandonados, com menos solidão e menos desprezados, pelo convívio entre eles próprios, com seus colegas e também com seus professores. Uma hipótese para este achado é o fato de os idosos que buscam estes grupos de convivência o fazerem devido à presença de menor sintomatologia depressiva, que leva a uma maior participação destes indivíduos nestes grupos.

No presente estudo, uma possível explicação para a ausência de sintomatologia depressiva na amostra estudada poderia estar relacionada com o trabalho realizado no grupo de convivência, que tem como objetivo principal a valorização do indivíduo idoso, enquanto ser pensante ativo e participante da sociedade. Segundo Castro (1998), em grupo, os idosos buscam e decidem sobre as motivações da própria vida, repensam e reformulam a própria história, liberando e usando produtivamente os recursos de sua personalidade. Dessa forma, o grupo atuaria como um possível fator de proteção contra a depressão na velhice.

Outra possibilidade de explicação para este achado estaria nas características sociodemográficas da amostra estudada. A amostra foi composta, em sua maioria, por idosas com nível de escolaridade elevado. Essa característica poderia explicar o baixo escore total na Escala de Depressão Geriátrica. No estudo de Trentini (2004), a escolaridade foi a variável mais consistentemente associada com a intensidade de depressão. Essa associação ocorre de forma inversa: quanto maior a escolaridade, menor a intensidade de sintomas psicológicos ou de somatizações. Desta forma, a escolaridade exerce um papel protetor para sintomas depressivos ou para sua expressão. Outros estudos também têm demonstrado uma relação entre menor escolaridade e maior número de idosos deprimidos (Cacciatore, 1998; Leite, Carvalho, Barreto \& Falcão, 2006).

A partir de uma comparação entre os clusters dos fatores de personalidade (IFP), diferentes domínios de qualidade de vida (WHOQOL-bref) e a variável depressão (GDS), observou-se que as idosas agrupadas no cluster 2, que são mais voltadas para si próprias, menos interativas e pouco dominantes, apresentaram escores menores de sintomas sugestivos de depressão em relação àquelas do cluster 1 , que são mais voltadas e interessadas no outro, mais deferentes, organizadas, persistentes e interativas. Embora os grupos que compõem o cluster 1 e o cluster 2 tenham diferido quanto aos escores totais na GDS, ambos os grupos não preencheram critérios sugestivos para a presença de sintomatologia depressiva. Este achado demonstra não haver um impacto clinicamente relevante nestes grupos, pois os dois clusters apresentaram ausência de sintomatologia depressiva. Uma associação entre dimensões de personalidade e depressão tem sido encontrada em diversos estudos (Costa et al., 1987; Costa et al., 2000; Diener \& Diener, 1996; Martin et al., 2002; Small et al., 2003; Watson \& Walker, 1996).

Entre as possíveis limitações deste estudo cabe ressaltar que ele foi conduzido com uma amostra com características relativamente homogêneas, apresentando nível de escolaridade elevado e boa situação socioeconômica, o que pode não refletir a realidade dos idosos em geral; entretanto, conhecer e entender as relações entre aspectos de personalidade, qualidade de vida e depressão pode ter implicações no planejamento e avaliação de serviços de saúde, bem como na implementação de oportunidades educacionais para este grupo populacional.

Estudos na literatura ainda são incipientes sobre aspectos de personalidade em idosos e sua relação com outras variáveis. Desta forma, outros estudos são sugeridos a fim de explorar a influência dos aspectos de personalidade sobre a qualidade de vida e depressão em idosos, bem como variáveis de impacto e interferência nesta associação.

\section{REFERÊNCIAS}

Bassanezi, C. (1997). Mulheres dos anos dourados. In M. Del Priore (Ed.), História das mulheres no Brasil (pp. 607639). São Paulo, SP: Contexto.

Cacciatore, F. (1998). Morbidity patterns in aged population in Southern Italy: A survey sampling. Archives of Gerontology and Geriatrics, 26, 201-13.

Cachioni, M. (1998). Envelhecimento bem-sucedido $e$ a participação numa universidade para a terceira idade: A experiência dos alunos da Universidade São Francisco. Dissertação de Mestrado não-publicada, Universidade Estadual de Campinas, Campinas, SP, Brasil.

Carpenito, L. J. (1995). Nursing diagnosis: Application to clinical practice. New York: Lippincott. 
Castro, O. P. (1998). O processo grupal, a subjetividade e a ressignificação da velhice. In O. P. Castro (Ed.), Velhice que idade é esta? Uma construção psicossocial do envelhecimento (pp. 35-55). Porto Alegre, RS: Síntese.

Costa, P. T., Herbst, J. H., McCrae, R. R., \& Siegler, I. C. (2000). Personality at midlife: Stability, intrinsic maturation and response to life events. Assessment, 7, 36578.

Costa, P. T., McCrae R. R., \& Zonderman, A. B. (1987). Environmental and dispositional influences on well-being: Longitudinal follow-up of American national sample. British Journal of Psychology, 78, 299-306.

Diener, E., \& Diener, C. (1996). Most people are happy. Psychological Science, 7, 181-185.

Erbolato, R. M. P. L. (1996). Universidade da terceira idade: Avaliação e perspectivas de alunos e ex-alunos. Dissertação de Mestrado não-publicada, Pontifícia Universidade Católica de Campinas, Campinas, SP, Brasil.

Farran, C. J., Herth, K. A., \& Popovich, J. M. (1995). Hope and hopelessness: Critical clinical constructs. Thousand Oaks, CA: Sage.

Field, D., \& Millsap, R. E. (1991). Personality in advanced old age: Continuity or change? Journal of Gerontology: Psychological Sciences, 46, 299-308.

Fleck, M. P. A., Leal, O. F., Louzada, S., Xavier, M., Chachamovich, E., Vieira, G., Santos, L., \& Pinzon, V. (1999). Desenvolvimento da versão em português do instrumento de avaliação de qualidade de vida da Organização Mundial da Saúde (WHOQOL -100). Revista Brasileira de Psiquiatria, 21, 19-28.

Hall, C. S., Lindzey, G., \& Campbell, J. B. (2000). Teorias $d a$ Personalidade. Porto Alegre, RS: Artes Médicas Sul.

Helson, R., Jones, C. J., \& Kwan, V. S. Y. (2002). Personality change over 40 years of adulthood: HLM analyses of two longitudinal samples. Journal of Personality and Social Psychology, 83, 752-766.

Herbst, J. H., McCrae, R.R., Costa Jr., P. T., Feaganes, J. R., \& Siegler, I. C. (2000). Self-perceptions of stability and change in personality at midlife: The UNC alumni heart study. Assessment, 7, 379-388.

Herman, H., Patrick, D. L., Diehr, P., Martin, M. L., Fleck, M., Simon, G. E., \& Buesching, D. P. (2002, July). Longitudinal investigation of depression outcomes in primary care in six countries: The LIDO study. Functional status, health service use and treatment of people with depressive symptoms. Psychological medicine, 32, 889902.

Kahn, R. L., \& Thomas, J. F. (2002). Well-Being: Concepts and Measures. Journal of Social Issues, 58, 627-44.

Kuehner, C. (2002). Subjective quality of life: Validity issues with depressed patients. Acta Psychiatric Scandinavia, 106, 62-70.

Labouvie-Vief, G., Diehl, M., Tarnowski, A., \& Shen, J. (2000). Age differences in adult personality: Findings from the United States and China. Journal of Gerontology, 1, 417.
Leite, V. M. M., Carvalho, E. M. F. C., Barreto, K. M. L., \& Falcão, I. V. (2006). Depressão e envelhecimento: Estudo nos participantes do Programa Universidade Aberta à Terceira Idade. Revista Brasileira de Saúde Materno Infantil, 6, 31-8.

Loures, M. C., \& Gomes, L. (2006). Prevalência da depressão entre os alunos da universidade aberta à terceira idade, Universidade Católica de Goiás no início e no término de seu curso. In V. P. Faleiros, \& A. M. L. Loureiro (Eds.), Desafios do envelhecimento: Vez, sentido e voz. (pp. 13958). Brasília, DF: Universa.

Maiden, R. J., Peterson, A. S., Caya, M., \& Hayslip, B., Jr. (2003). Personality Changes in the Old-Old: A Longitudinal Study. Journal of Adult Development, 10, 3139.

Martin, P., Valora, L. M., \& Poon, L. W. (2002). Age change and differences in personality traits and states of the old and very old. Journal of Gerontology: Psychological Sciences, $57,144-52$.

McAdams, D. P. (1995). What do we know when we know a person? Journal of Personality, 63, 365-96.

McCrae, R. R., \& Costa, P. T., Jr. (1987). Validation of fivefactor model of personality across instruments and observers. Journal of Personality and Social Psychology, $52,81-90$.

McCrae, R. R., \& Costa, P. T., Jr. (1994). The stability of personality: Observations and evaluations. Current Directions in Psychological Science, 3, 173-175.

Neri, A. L. (2000). Qualidade madura no atendimento domiciliário. In Y. A. O. Duarte, \& M. J. D. Diogo (Eds.), Atendimento domiciliar: Um enfoque gerontológico (pp. 33-47). São Paulo, SP: Atheneu.

Neri, A. L. (2001). Velhice e qualidade de vida na mulher. In A. L. Neri (Ed.), Desenvolvimento e envelhecimento: Perspectivas biológicas, psicológicas e sociológicas (pp. 161-200). Campinas, SP: Papirus.

Neugarten, B. L., Moore, J. W., \& Lowe, J. (1965). Age norms, age constraints, and adult socialization. American Journal of Sociology, 70, 710-717.

Papalia, D. E., \& Olds, S. W. (2000). Desenvolvimento Humano. Porto Alegre, RS: Artmed.

Pasquali, L., Azevedo, M. M., \& Ghesti, I. (1997). Inventário Fatorial de Personalidade: Manual técnico e de avaliação. São Paulo, SP: Casa do Psicólogo.

Ruth, J. E., \& Coleman, P. (1996). Personality and aging: Coping and management of the self in later life. In J.E. Birren \& K.W. Schaie (Eds.), Handbook of the Psychology of aging (pp. 308-322). San Diego, CA: Academic Press.

Ryff, C. D. (1991). Possible selves in adulthood and old age: A tale of shifting horizons. Psychology and Aging, 6, 286-95.

Schaie, K. W., \& Willis, S. L. (1991). Adult personality and psychomotor performance: Cross-sectional and longitudinal analysis. Journal of Gerontology, 46, 257-84.

Sheikh, J. I., \& Yesavage, J. A. (1986). Geriatric depression scale (GDS): Recent evidence and development of a shorter version. Clinical Gerontology, 5, 165-73. 
Silva, F. P. (1999). Motivos para frequentar universidade da terceira idade, crenças em relação à velhice e bem-estar subjetivo. Dissertação de Mestrado não-publicada, Universidade Estadual de Campinas, Campinas, SP, Brasil.

Small, B. J., Herzog, C. H., Hultsch, D. F., \& Dixon, R. A. (2003). Stability and change in adult personality over 6 years: findings from the Victoria Longitudinal Study. Journal of Gerontology: Psychological Sciences, 58, 16676.

Staudinger, U. M., Marsiske, M., \& Baltes, P. B. (1993). Resilience and levels of reserve capacity in later adulthood: Perspectives from life-span theory. Development and Psychology, 5, 541-66.

Steunenberg, B., Beekman, A. T. F., Deeg, D. J. H., \& Kerkhof, A. J. F. M. (2006). Personality and the onset of depression in late life. Journal of Affective Disorders, 92, 243-51.

Tavares, A. (2005). Compêndio de Neuropsiquiatria Geriátrica. Rio de Janeiro, RJ: Guanabara Koogan.

The WHOQOL group. (1998). The World Health Organization quality of life assessment (WHOQOL): development and general psychometric properties. Social Science and Medicine, 46, 1569-85.

Trentini, C. M. (2004). Qualidade de vida em idosos. Tese de Doutorado não-publicada, Universidade Federal do Rio Grande do Sul, Porto Alegre, RS, Brasil.

Xavier, F. M., Ferraz, M. P., Argimon, I., Trentini, C. M., Poyares, D., Bertollucci, P. H., Bisol, L. W., \& Moriguchi, E. H. (2002). The DSM-IV 'minor depression' disorder in the oldest-old: Prevalence rate, sleep patterns, memory function and quality of life in elderly people of Italian descent in Southern Brazil. International Journal of Geriatric Psychiatry, 17, 107-16.

Watson, D., \& Walker, L.M. (1996). The long-term stability and predictive validity of trait measures of affect. Journal of Personality and Social Psychology, 70, 567-77.

Recebido em 05/07/2007

Aceito em 15/02/2009

Endereço para correspondência : Tatiana Quarti Irigaray. Av. João Pessoa, no 809, sala 103, Cidade Baixa, CEP 90040-000, Porto Alegre, Rio Grande do Sul, Brasil. E-mail: tatiana.irigaray@ superig.com.br 